\title{
BEMA statt GOZ? Zweimal das grundsätzlich gleiche Problem
}

\begin{abstract}
Der erste Problemfall tritt gelegentlich auf bei Privatleistungen, insbesondere Funktionsabformung im "stark reduzierten Restgebiss". Es liegt ein Restgebiss mit vier Zähnen im Unterkiefer und einseitig sehr ausgedehnter Freiendsituation vor. Es erfolgte die gleichartige prothetische Versorgung eines GKV-Patienten. Die private Zusatzversicherung lehnt Kostenübernahme einiger privat berechneter GOZ-Leistungen wie 0060 (Diagnostik-/ Planungsmodelle), 4040 (okklusal-artikuläres Grobeinschleifen), 4070/4075 (chirurgische geschlossene PAR-Therapie) und 4080 (Gingivoplastik) ab, da es sich um originäre GKV-Leistungen handele, für die sie vertragsgemäß nicht zahlungspflichtig sei.
\end{abstract}

Die Argumentation der Zusatzversicherung erscheint auch aus Gründen außerhalb des Tarifs zutreffend: Diagnostik-/Planungsmodelle werden nach Ziffer $7 \mathrm{~b}$ und Einschleifen nach Ziffer 89 Bema abgerechnet. Der GKV-Patient hat auch einen Rechtsanspruch auf PAR-Behandlung in der GKV (P200, P201 etc.), und auf Teil 2 des Heil- und Kostenplans gehören Zahnersatzleistungen, keine PAR-Behandlungsmaßnahmen (die zudem richtliniengemäß vorher erfolgt sein sollen).

Die private Zusatzversicherung lehnt zusätzlich die Erstattung für die Nr. 5190 GOZ (Funktionsabformung Unterkiefer) $\mathrm{ab}$ und argumentiert auch hier, dass der GKV-Patient einen Anspruch auf die GKV-Leistung nach Ziffer 98c Bema hätte. Das verwundert. In der GKV-Abrechnungsbestimmungen zur Ziffer $98 \mathrm{c}$ heißt es nämlich:

„Leistungen nach Nr. 98c sind bei zahnlosem Kiefer und bei stark reduziertem Restgebiss - in der Regel bis zu drei Zähnen - abrechnungsfähig."

Diese Formulierung gibt jedoch der Zusatzversicherung Recht: Im begründeten Ausnahmefall (hier extrem langer einseitiger Freiendsattel) ist selbst bei einem Restgebiss mit vier Zähnen in einem Kiefer in der GKV-Zahnersatzversorgung die Ziffer $98 \mathrm{c}$ ansetzbar (Sonderregel). Ansonsten hätte man nicht die Formulierung "in der Regel" gewählt, sondern rigoros formuliert ,... sind nur bei stark reduziertem Restgebiss bis zu drei Zähnen abrechnungsfähig“.

Ziemlich sicher ist eine Abrechnung unzulässig, die sowohl die Ziffer 98c Bema auf Teil 1 des GKV-Plans aufführt und auf Teil 2 die Nr. 5190 GOZ: Das ist in aller Regel unzulässige Doppelabrechnung.

Der zweite Problemfall tritt auf bei der GKV-Wurzelkanalbehandlung mit der Fragestellung:

\section{Vierte Medikamentöse Einlage nach Bema oder privat?}

In der GKV-Leistungsbeschreibung der Nr. 34 (Med) heißt es „Medikamentöse Einlage ... je Zahn und Sitzung“. In der offiziellen Abrechnungsbestimmungen dazu findet sich: „Medikamentöse Einlagen sind grundsätzlich auf drei Sitzungen beschränkt.“"
In nichtamtlichen Verlautbarungen heißt es wiederholt, somit sei die nötige vierte Einlage in den betreffenden Zahn privat nach Nr. $2340 \mathrm{GOZ}$ zu berechnen.

Das ist einmal aus formal-gebührentechnischen Gründen nicht richtig, denn Nr. $2340 \mathrm{GOZ}$ (medikamentöse Einlage) ist nur in Verbindung mit den GOZ-Nrn. 2360, 2380 und 2410 ansatzfähig, nicht in Verbindung lediglich mit Bema-Ziffern.

Die 4. Einlage im selben Zahn privat anzusetzen, ist aber auch gemäß GKV-Abrechnungsbestimmung nicht richtig. Dort steht "grundsätzlich auf drei Sitzungen beschränkt". Das heißt gemäß juristischem Sprachgebrauch gegebenenfalls im begründeten Sonderfall auch in einer 4. Sitzung als 34 (Med) zu berechnen, falls zahnmedizinisch erforderlich. Das kann zum Beispiel bei einem akzidentellen Re-Infekt etcetera der Fall sein. Der GKVPatient hat dann einen Anspruch auf die vierte GKV-Leistung, die kann ihm nicht einfach mittels Privatberechnung vorenthalten werden.

Selbstverständlich kann der GKV-Patient eine insgesamt private "Wurzelbehandlung" mittels schriftlicher Vereinbarung nach $₫ 7$ (7) EKV-Z bzw. § 4 (5) BMV-Z wählen statt der GKVBehandlung. Dann gibt es allerdings keine Kostenbeteiligung der GKV-Kasse. Es existieren Sondervereinbarungen bzw. Sonderverträge zur Wurzelkanalbehandlung in bestimmten KZVBereichen mit einer Art Mehrkostenregelung.

C ZA 2016, Dr. P. Esser

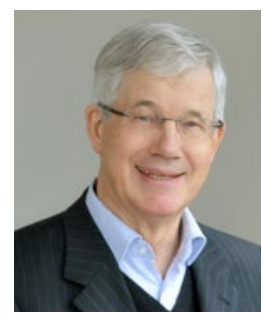

Dr. Peter H.G. Esser

GOZ-Experte und -Berater der ZA eG www.za-abrechnung.de 